\title{
CLASSIFICATION OF LAND-COVER THROUGH MACHINE LEARNING ALGORITHMS FOR FUSION OF SENTINEL-2A AND PLANETSCOPE IMAGERY
}

\author{
Maycol Alejandro Zaraza Aguilera ${ }^{1}$ \\ ${ }^{1}$ University Distrital Francisco Jose de Caldas, Master in Information and Communications Sciences - Engineering Faculty-, \\ Bogota, Colombia - mazarazaa@correo.udistrital.edu.co
}

KEY WORDS: Land-cover classification, Machine Learning, Sentinel-2A, PlanetScope, Fusion Images

\begin{abstract}
:
To monitor and manage the changes in the land use and land cover, is vital the process of classification; machine learning offers the potential for effective and efficient classification of remotely sensed imagery. However, not many articles have explicitly dealt with the effects of image fusion on land-cover classification accuracy. Although some studies have compared thematic mapping accuracy produced using different classification algorithms, there are no currently many studies that utilize image fusion for assessing different machine learning algorithms for classification purposes. The main aim of this study is to compare different machine learning algorithm for pixel classification of imagery fused with sensors Sentinel-2A and PlanetScope. The method used for image fusion is a variational model, the high spectral resolution of Sentinel-2A imagery and the high spatial resolution of PlanetScope imagery was fused; the machine learning algorithms evaluated are six that have been widely used in the remote sensing community: DT (Decision Tree), Boosted DT, RF (Random Forest), SVM radial base (Support Vector Machine), ANN (Artificial Neural Networks), KNN (k-Nearest Neighbors), for the classification four spectral indices (NDVI, NDMI, NDBI, MSAVI) were included, derived of the image fusion. The results show that the highest accuracy was produced by SVM radial base (OA: 87.8\%, Kappa: 87\%) respect to the other methods, nevertheless the methods RF, Boosted DT and KNN shown to be very powerful methods for classification of the study area.
\end{abstract}

\section{INTRODUCTION}

The classification for land use and land cover (LULC) constitutes a key variable for the monitoring earth that in general have shown a close correlation with human activities and the physical environment, the fast development of human societies has intensified different types of activities that have resulted in a continuous and noticeable influence on LULC, (Petropoulos, Partsinevelos, \& Mitraka, 2013).

In the last years remote sensing scientists are increasingly adopting machine learning classification algorithms for LCLU mapping, (Shih, Stow, \& Tsai, 2018). Compared with products derived by traditional statistical classifiers (e.g. minimum distance, maximum likelihood, and parallelepiped classifiers), previous research results generally show that machine learning classifiers yield more accurate and reliable products, particularly if abundant training data are available, (Schneider, 2012). While unsupervised classifiers is based on similarity measures like color information, distance between neighboring pixels, etc., this may not be accurate with the reality, (Sivagami, Krishankumar, \& Ravichandran, 2018).

Machine learning offers the potential for effective and efficient classification of remotely sensed imagery. The strengths of machine learning include the capacity to handle data of high dimensionality and to map classes with very complex characteristics, it can accept a variety of input predictor data, and do not make assumptions about the data distribution (i.e. are nonparametric), (Maxwell, Warner, \& Fang, 2018).

A wide range of studies have generally found that these methods tend to produce highs accuracy, authors like (Keshtkar, Voigt, \& Alizadeh, 2017) compared pixel-based random forest
(RF) and decision tree (DT) classifier methods and a support vector machine (SVM) algorithms both in pixel-based and object-based approaches for classification of land-cover in a heterogeneous landscape with Landsat imagery, they found that the object-based SVM classifier is the most accurate with a kappa value of 0.88 .

(Maxwell et al., 2018) reviewed and applied six types of machine learning classifiers (i.e. support vector machine, decision tree, random forest, artificial neural network, boosted decision tree, and k-nearest neighbors) for two datasets of AVIRIS imagery. The map derived from SVM (94.4\%), boosted DT $(87.6 \%)$ and RF (87.8\%) classifiers were found to have the highest overall accuracy for each dataset.

(Shih et al., 2018) established a comparison of machine learning classifiers for Landsat-based land cover and land use mapping, they employed ANN, DT, SVM, and RF classifiers to identify optimal polynomial degree of input features and hyperparameters with Landsat imagery of a region in China and Ghana and obtained that SVM has the highest overall accuracy (72.6\%). (Abdi, 2019) compared the classification performance of four non-parametric algorithms: support vector machines (SVM), random forests (RF), extreme gradient boosting (Xgboost), and deep learning (DL) in a boreal landscape using Sentinel-2 data, where the four tested algorithms produced similar overall accuracies ranging between 0.733 to 0.758 .

While (Sivagami et al., 2018) analyzed the performance of different algorithms (Gaussian SVM, KNN, Decision Tree and Ensemble) for labelling each pixel of a orthophoto image, concluded that the fine Gaussian support vector machine outperforms the other techniques with an overall accuracy of 75.15\%; and (Petropoulos et al., 2013) proposed a 
methodology for quantifying the impact of surface mining activity and reclamation from a watershed to local scale. The method is based on a Support Vector Machines (SVMs) classifier combined with multi-temporal change detection of Landsat TM imagery, the overall classification accuracy of the thematic land cover maps produced was reported over $90 \%$.

On the other hand recent improvements in satellite imaging technology have given rise to new opportunities for more accurate land use and land cover mapping; different Earthobserving satellites, such as Sentinel-2A and PlanetScope with different spatial resolutions and with global almost daily revisit time provide valuable information about the earth surface in a short time, (M. Gašparović, Medak, Pilaš, Jurjević, \& Balenović, 2018).

Image fusion is a process of combining images obtained by sensors of different wavelengths viewing the same scene, to form a composite image with improved image content that makes it easier for the user to detect, recognize and identify targets, (Wald, 1999). Specifically, as far as spatial and spectral resolution are concerned, high spectral resolution can generally be helpful in discriminating LULC types, while high spatial resolution may hold advantages in identifying terrain features and structures, (Tso \& Mather, 2016). In this research the high spectral resolution of Sentinel-2A imagery and the high spatial resolution of PlanetScope imagery was fused.

(Ballester, Caselles, Igual, Verdera, \& Rougé, 2006) has proposed an algorithm based on the assumption that, to a large extent, the geometry of the spectral channels is contained in the topographic map of its panchromatic image, called a variational model for $\mathrm{P}+\mathrm{XS}$ image fusion. This have been evaluated in some researches like (He, Condat, Chanussot, \& Xia, 2012) where experimental results on IKONOS satellite images demonstrated the effectiveness of the variational model, also (Mateo Gašparović \& Jogun, 2017) evaluated this model for Sentinel-2A imagery, the results showed that the fusion method, $\mathrm{P}+\mathrm{XS}$, improved the overall land cover classification accuracy.

While (M. Gašparović et al., 2018) evaluated a fusion of Sentinel-2 imagery with PlanetScope because of its higher spatial resolution, the fusion method validation was provided based on the land-cover classification accuracy, the classification was made with random forest (RF) classifier, results show better accuracy for fused imagery than the Sentinel-2 imagery.

Actually, it is vital to map the LCLU at the landscape scale in order to monitor and manage the changes in the earth, classification using satellite data provides a crucial starting point for this endeavor, (Abdi, 2019). Therefore, more research is required in this area (especially for preparation of land-cover maps) to ascertain the superiority of one method or group of methods over others. However, not many articles have explicitly dealt with the effects of image fusion on land-cover classification accuracy, although some studies have compared thematic mapping accuracy produced using different classification algorithms, there are no currently many studies that utilize image fusion for assessing different machine learning algorithms for classification purposes.

The main aim of this paper is to compare different machine learning algorithm for pixel classification of imagery fused from sensors Sentinel-2A and PlanetScope. The method used for image fusion is a variational model for $\mathrm{P}+\mathrm{XS}$; the machine learning algorithms evaluated are six that have been widely used in the remote sensing community: DT (Decision Tree), Boosted DT, RF (Random Forest), SVM radial base (Support Vector Machine), ANN (Artificial Neural Networks), KNN (kNearest Neighbors).

\section{STUDY AREA AND DATASET}

\subsection{Study area}

The study area of this research is a part of the region of the mine Cerrejon, this is ubicated in middle of five municipalities (Riohacha, Hato Nuevo, Albana, Maicao and Barrancas), (Figure 1) all belonging to the department of Guajira in Colombia, this mine have an open-pit exploitation, there extracts coal. The study zone has an area of 207.75 square $\mathrm{km}$ or $20775.49 \mathrm{Ha}$, and there are land covers like forest, grass, bushes, urban area, water, bare soil and mining. The six machine learning algorithms were evaluated in this area.

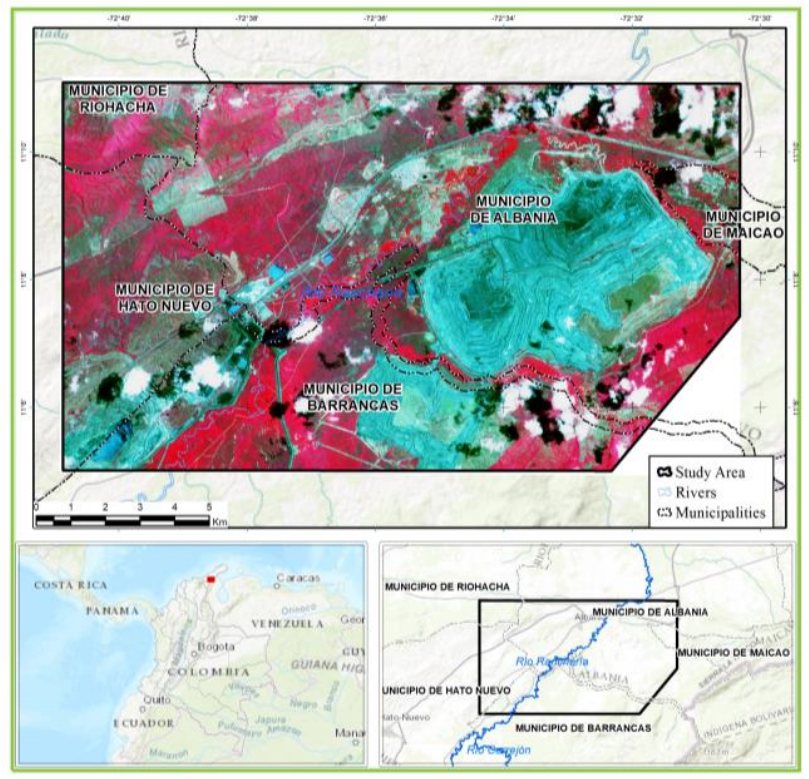

Figure 1: Study Area located in Guajira, Colombia

\subsection{Dataset}

In this research, satellite image Sentinel-2A of 10-m and 20-m spatial resolution and 3-m satellite image PlanetScope were used (Fig. 2). Sentinel-2A (S-2A) is developed by the European Space Agency specifically for the Copernicus program covering all of Earth's landmass every 5 days. It has an optical multispectral instrument with 13 spectral bands: four bands at $10 \mathrm{~m}$ spatial resolution, six bands at $20 \mathrm{~m}$ spatial resolution, and three bands at $60 \mathrm{~m}$ spatial resolution, (Copernicus, 2017). The product used was Level-2A that provide Bottom of Atmosphere (BOA) reflectance, images are ortho-images in UTM/WGS84 projection.

Planet operates with more than 175 PS satellites that collect multispectral imagery in 4 bands with a collection capacity of 300 million square km per day, ability to image all of Earth's landmass every day (PlanetLabs, 2019). The product used was 4-band SR (Surface Reflectance) analytic data with a spatial resolution resampled at 3 meters and projected in the UTM projection using the WGS84 datum, this product is orthorectified, the image of 31th July 2019 was used. For this research, for S-2A four bands $10-\mathrm{m}(2,3,4,8)$ and six bands 
20-m Red Edge (RE; 5, 6, 7, 8a) and SWIR (Bands 11,12) for the date 2th August 2019 was used.

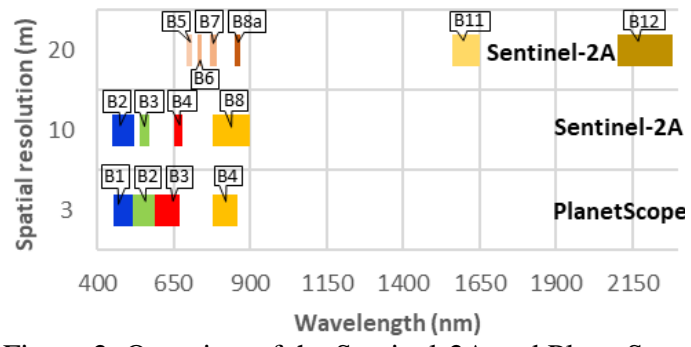

Figure 2: Overview of the Sentinel-2A and PlanetScope spectral bands employed. Adapted of (M. Gašparović et al., 2018).

\section{METHODOLOGY}

The methodology was divided in three phases, the first phase was download and clipping of the Sentinel-2A (S-2A) and PlanetScope (PS) imagery, this was followed by the phase of Image Fusion between the two images and the assessment process, the third phase correspond to classification land-cover of the study area through machine learning algorithms including spectral indices and image fused. The Figure 3 present the workflow employed in this research.

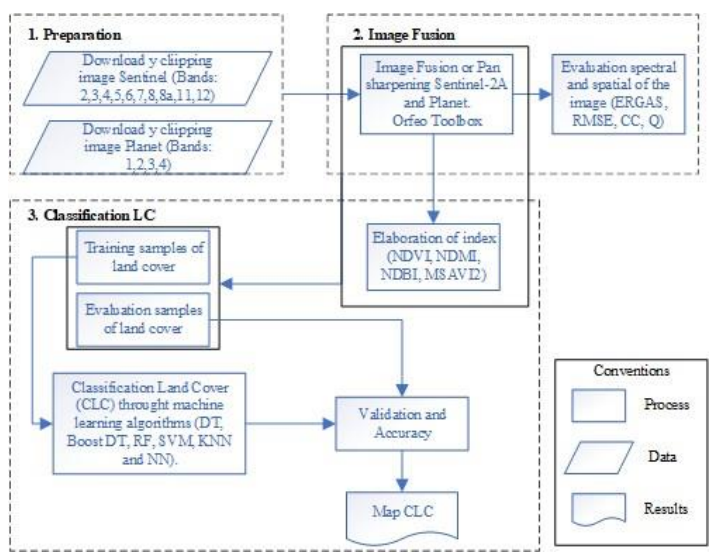

Figure 3: Workflow followed in the present research

\subsection{Preparation}

The Sentinel-2A and PlanetScope imagery were download and clipped to the study area, both images were georeferenced in the WGS84-UTM18N coordinate system.

\subsection{Image Fusion}

The method used for the image fusion between Sentinel-2A and Planetscope imagery is based in (Mateo Gašparović \& Jogun, 2017), they used variational $(P+X S)$ fusion method. The $P+$ $\mathrm{XS}$ method introduces the geometry information of the higher resolution image by aligning all edges of the higher resolution image with each lower resolution multispectral band. To obtain the spectral information for the fused image, the method assumes that images taken in different spectral bands share common geometric information and that the higher resolution image can be approximated as a linear combination of the highresolution multispectral bands (Ballester et al., 2006; Mateo Gašparović \& Jogun, 2017; He et al., 2012).

Based on the research of (M. Gašparović et al., 2018), each band S-2A, was fused with a band PS with similar spectral characteristics; therefore, $10-\mathrm{m} \mathrm{S}-2$ bands $(2,3,4,8)$ are fused based on the PS bands 1,2,3,4 respectively. The band 8A, S-2A $20 \mathrm{~m}$ was fused with the PS band 4 . For the bands 5,6,7,11,12 and based in (M. Gašparović et al., 2018) this were fused with the synthetized band $(\mathrm{S})$ given by the equation 1 .

$$
B_{s}=\frac{B_{2}+B_{4}}{2}
$$

where $B_{3}$ and $B_{4}$ represent PS band 3 and 4, respectively. Image fusion process was made with the use of open-source software Orfeo Toolbox (OTB) version 6.6.1. OTB algorithm for image fusion was accessed from Monteverdi.

For the spectral and spatial evaluation of the image fusion we used the traditional quality metrics like ERGAS (Alparone et al., 2007), RMSE (Root-Mean-Square Error) (Otazu, Gonzalez-Audicana, Fors, \& Nunez, 2005), CC (Correlation Coeficient) (Nunez et al., 1999), and Index of Quality Universal (Q) (Wang \& Bovik, 2002). The evaluation of results in image fusion traditionally consist in to calculate the similarity between the merged image and a reference image, however, still there is not universally accepted standard index to evaluate image fusion processes (Renza Torres, 2010).

\subsection{Classification Land-Cover (CLC)}

Obtained the image through the image fusion, a process of classification land-cover was developed using machine learning algorithms, the first step is the elaboration and generation of four spectral indices derived from the resultant bands of image fusion, the indices chosen let to provide useful information for distinguish between different level of vegetation and soil. These indices help to improve classification models; they are combined with spectral variables derived from the image fusion which allow better spectral differentiation of interest classes.

The spectral indices employed (Table 1) are: Normalized Difference Vegetation Index (NDVI) since vegetation cover is generally regarded as a key indicator of restoration success reflecting the critical stages of ecosystem development and functionality, (Vallauri, Aronson, Dudley, \& Vallejo, 2005); Normalized Difference Built-up Index (NDBI) uses the SWIR band to identify artificial surfaces because of their strong reflectivity in that portion while using the NIR to suppress the influence of vegetated surfaces (Zha, Gao, \& Ni, 2003).

Normalized Difference Moisture Index (NDMI), the difference between the NIR and the MIR appears to be the ability of the MIR wavelengths to absorb water, in a green leaf, the NIR band has the maximum reflectance and the reduction of reflectance of the MIR as compared to the NIR is due to the absorption of water (Wilson \& Sader, 2002) and MSAVI (Modified SoilAdjusted Vegetation Index) it show to increase the dynamic range of the vegetation signal while further minimizing the soil background influences, resulting in greater vegetation sensitivity as defined by a "vegetation signal" to "soil noise" ratio, it is more applicable than SAVI in practice because soil effects are implicitly adjusted according to different vegetation densities (Qi, Chehbouni, Huete, Kerr, \& Sorooshian, 1994; Wu, Wang, \& Bauer, 2007).

\begin{tabular}{|c|}
\hline INDEX \\
\hline NDVI $=($ NIR - RED $) /($ NIR + RED $)$ \\
\hline NDMI $=($ NIR - SWIRI $) /($ NIR + SWIR 1$)$ \\
\hline MSAVI $=$ \\
\hline$\frac{2\left(R E 8_{a}\right)+1-\sqrt{\left(2\left(R E 8_{a}\right)+1\right)^{2}-8\left(R E 8_{a}-\text { RE5 }\right)}}{2}$ \\
\hline
\end{tabular}

Table 1: Spectral indices used 
Training data is a crucial component in supervised learning and most machine learning algorithms require a large number of training data samples, (Abdi, 2019). Sample size and quality of training data have generally showed to have a great impact on the accuracy of the classification, training data may even have a greater impact than the algorithm used, (Maxwell et al., 2018). A total of 200 training points was collected for all the ten classes (Forest, Grasses, Water, Bushes, Bare soil, Mining, Urban area o Built-up, Secondary Forest, Clouds, Shadows) in the study area, using random sampling from the image fusion and spectral indices. Around for each point a kernel of 10 by 10 pixels was created for increase the sample size and to obtain 20.000 pixels or samples, the training set was divided in $30 \%$ for validation and $70 \%$ for training.

The statistical variations of each class are analyzed and it is determined whether the class should be separated into different subclasses (cluster), seeking to improve the identification of spectral variations within it.

According to (Bradski \& Kaehler, 2013; Tso \& Mather, 2016) the most used Machine Learning algorithms are: Vector support machines (SVM), Decision trees (DT) and Random Forests (RF). Each of these non-parametric learning classifiers have different sets of features or parameters, such as the ability to manage missing measurements or categorical input variables. However, there is not "best" classifier, averaging over all possible types of data distributions, all classifiers would generate the same result, (Bradski \& Kaehler, 2013).

Based on the above, we employed six different machine learning algorithms for the process of classification: Support Vector Machine (SVM), Decision Trees (DT), Random Forest (RF), Boosted DT, artificial neural networks (ANN) and knearest neighbors $(\mathrm{k}-\mathrm{NN})$, which in the different methodological reviews showed good thematic accuracy, (Abdi, 2019; Keshtkar et al., 2017; Maxwell et al., 2018). The classifications were carried out using the free statistical software tool R, within R, we used the caret package, (Kuhn et al., 2019), which provides a standard syntax to execute a variety of machine-learning methods, thus simplifying the process of comparing different algorithms and approaches. Other software tools, such as scikit-learn for Python and Weka, could also potentially be used.

This part provides a brief description and introduction of the algorithms used in the classification.

Support Vector Machines (SVM): It is an image classification alternative, which allows to obtain exact classifications from a set of reduced training samples (Addesso, Conte, Longo, Restaino, \& Vivone, 2012; I. Lizarazo, 2008). The main attraction of SVM is its ability to minimize classification errors, creating a hyperplane between each pair of classes, that maximizes the distance between the support vectors of each class (Buddhiraju \& Rizvi, 2010; Tso \& Mather, 2016). If it is not possible to construct that hyperplane in the original spectral space, the separation is carried out in a spectral space of higher dimension, (Addesso et al., 2012).

The projection to a higher dimensionality is known as the kernel trick. There are many possible kernels (Kavzoglu \& Colkesen, 2009), and each kernel may have a different set of required user-specified parameters. Common kernels used in remote sensing are polynomial kernels and the radial basis function (RBF) kernel. In this research we used (RBF) kernel.
Decision Trees (DT): The DT algorithm is a technique that constructs a series of rules based on the values of the attributes of a training sample, to assign a class to each of the objects of interest through an objective function, the quality is informally informed as a model of classification, (Tan, Steinbach, Karpatne, \& Kumar, 2018). The design of a DT is based on knowledge of the spectral properties of each class and of relationships among the classes, (Tso \& Mather, 2016).

Random Forest (RF): RF is an ensemble classifier, as it uses a large number of DTs in order to overcome the weaknesses of a single DT, (Maxwell et al., 2018). It is a classification technique that is based on the aggregation of a large number of decision trees (Boulesteix, Janitza, Kruppa, \& König, 2012), the combination of many trees is often more accurate that depend on just one tree, (Ivan Lizarazo, 2010).

Boosted DT: Boosted DTs are also an ensemble method using DTs. The basis of the boosting techniques is to assign weights to the individual elements of the training data set, (Tso \& Mather, 2016). Boosting enables multiple iterations of DT classifiers using a base algorithm, while modifying the training sample at each iteration to concentrate on those samples that were difficult to classify correctly in the previous iterations, each iteration generates a DT, with the final classifier being produced by a weighted vote, (Evrendilek \& Gulbeyaz, 2011).

Artificial Neural Networks (ANN): ANNs are algorithmic classifiers which simulate human neural synapses that conduct signals and transforms them into information, ANNs contain three types of layers, an input layer, an output layer, and hidden layer; each node in the output layer is a class, for example, different types of land cover in a study area, from each iteration of a training, each layer is slightly adjusted to generate a model that can separate most of the training samples into the correspondent classes, (Shih et al., 2018). The challenge with ANNs is that they can be slow to train, can produce non-optimal classifications, and are very easy to over-train, (Maxwell et al., 2018).

K-Nearest Neighbors (KNN): the classifier allocates unknown sample to the neighbors to which it is closest in feature space. An inverse distance weighting (IDW) function can be incorporated into the k-NN classifier to give more weight to information from a neighbor close to an unclassified observation than from a more distant neighbor, (Tang, Jing, Atkinson, \& Li, 2016).

Finally, through a parallelization and optimization process, machine learning algorithms are applied, the parallelization optimizes processing times and the optimization seeks the use of the best configuration parameters of the applied algorithms and the best classification performance, avoids that classifier underfitting or overfitting, (Shih et al., 2018).

The 30 percent of the training set was used for the validation process, the accuracy of each algorithm was assessed using a number of metrics derived from an error matrix, these include Overall Accuracy (OA), Producer's Accuracy (PA), and User's Accuracy (UA), (Olofsson et al., 2014), also the accuracy was assessed using the kappa coefficient, it is a measure of overall statistical agreement of an error matrix, which takes nondiagonal elements into account, (Jensen, 2005). The algorithms with a Kappa greater than $80 \%$ are selected as the best, while their correlation is less than $75 \%$. If there is no more than one 
method with Kappa greater than $80 \%$, those with a Kappa greater than $75 \%$ are chosen, while their correlation is less than 75\%. If there are no suitable methods, the algorithm with the highest Kappa index obtained is selected.

All maps are generated with a filtering by area, eliminating units smaller than 900 square meters and adding them to the predominant class around them.

\section{RESULTS AND DISCUSSION}

\subsection{Image Fusion}

The obtained results give evidence the good visual appearance and the spectral content preservation of fused image, through of the variational $(\mathrm{P}+\mathrm{XS})$ fusion method, the bands $2,3,4,5,6,7,8,8 \mathrm{a}, 11,12$ of Sentinel-2A to a spatial resolution of 3 meters (Figure 4) we obtained.

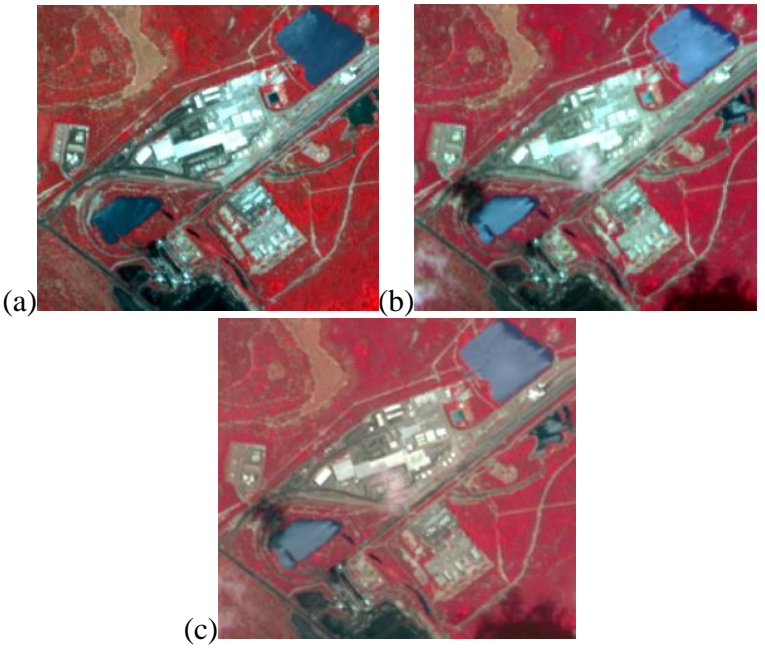

Figure 4: Results for a sub-area in composition "False Color": (a) PlanetScope (RGB: 4,3,2); (b) Sentinel-2A (RGB: 8,3,2); (c) Fused image (RGB: 8,3,2).

To examine the quality of the fused image, four indices were evaluated: ERGAS, RMSE, CC and Index of Quality Universal (Q) these were calculated for all bands (Table 2). For the spatial evaluation each band of the fused image was compared with the band Planetscope (PS) image employed in the process, while for the spectral evaluation each band of the fused image was compared with each band of the Sentinel-2A (S2A) image used.

For the spatial evaluation (Table 2), the index ERGAS indicate values between 2.2 and 8 where its ideal value is 0 , the best band is 4 , the bands with the highest values were the fused with the bands S2A 20m; for the index CC, its value ideal is 1 , all the bands have values between -0.5 and 0.4 this indicate that there are an inverse correlation in the bands 2,3,4 y 8 and a low correlation in the rest. The index $\mathrm{Q}$ is 0 for all the bands this indicate that spatially none band have high quality, for the index RMSE the lowest values are in the band 2,3,4 and 8 this indicate that these bands have the least spatial difference with the bands of the PS image. In conclusion spatially the bands fused with S2A $10 \mathrm{~m}(2,3,4,8)$ are better than bands fused with S2A $20 \mathrm{~m}$.

For the spectral evaluation (Table 2), the values of the indices CC $\mathrm{y} \mathrm{Q}$ are close to 1 , this indicate that there is a high correlation between the band fused and the bands of S2A image, for the bands 2,3,4 and 8 the values index ERGAS are higher than for the other bands, for the index RMSE all bands have values low this indicate that these have low spectral difference with the bands of the S2A image. In general, spectrally all the bands of fused image are good.

\begin{tabular}{|c|c|c|c|c|c|c|c|c|}
\hline & \multicolumn{4}{|c|}{ Spatial Evaluation } & \multicolumn{4}{c|}{ Spectral evaluation } \\
\cline { 2 - 9 } & ERGAS & CC & $\mathbf{Q}$ & RMSE & ERGAS & CC & $\mathbf{Q}$ & RMSE \\
\hline Ideal & $\mathbf{0}$ & $\mathbf{1}$ & $\mathbf{1}$ & $\mathbf{0}$ & $\mathbf{0}$ & $\mathbf{1}$ & $\mathbf{1}$ & $\mathbf{0}$ \\
\hline B2 & 7.354 & -0.52 & -0.01 & 1.54 & 9.989 & 0.995 & 0.995 & 1.412 \\
\hline B3 & 6.066 & -0.59 & -0.01 & 1.54 & 9.430 & 0.995 & 0.995 & 1.407 \\
\hline B4 & 5.475 & -0.53 & -0.01 & 1.54 & 9.445 & 0.995 & 0.995 & 1.409 \\
\hline B5 & 8.043 & 0.297 & 0 & 4.004 & 1.033 & 0.992 & 0.993 & 0.968 \\
\hline B6 & 8.043 & 0.406 & 0 & 4.004 & 0.809 & 0.992 & 0.992 & 1.112 \\
\hline B7 & 8.043 & 0.416 & 0 & 4.004 & 0.786 & 0.992 & 0.993 & 1.195 \\
\hline B8 & 2.281 & -0.66 & -0.04 & 1.54 & 7.188 & 0.995 & 0.995 & 1.386 \\
\hline B8a & 8.043 & 0.426 & 0 & 4.004 & 0.783 & 0.992 & 0.992 & 1.291 \\
\hline B11 & 8.043 & 0.424 & 0 & 4.004 & 0.756 & 0.993 & 0.992 & 1.127 \\
\hline B12 & 8.043 & 0.312 & 0 & 4.004 & 1.033 & 0.992 & 0.991 & 1.072 \\
\hline
\end{tabular}

Table 2: Quantitative analyses of the image fusion

\subsection{Classification Land-Cover (LC)}

Table 3 presents the overall accuracy (OA) results and the kappa coefficients for each classification method used. The smaller obtained OA is for the method ANN (18.3\%) and kappa coefficient of $12.8 \%$, likely because of the more limited training dataset, which suggests a modification to the parameters and training dataset for this algorithm, the methods Boosted DT, RF, SVM-Radial and KNN have an OA greater than $80 \%$ equally a kappa coefficient greater than $80 \%$, except to $\mathrm{KNN}$ $(79.3 \%)$. The correlation obtained between these is less than $75 \%$. The classification derived from SVM-Radial algorithm is the most accuracy (Kappa:87\%), followed by Boosted-DT and $\mathrm{RF}$, although the difference between these is quite small.

The high accuracy of SVM may be attributed to the classifier properties, SVMs are able to simultaneously minimize the empirical classification error and maximize the class separation using various transformations of the class separation hyperplane, (Petropoulos et al., 2013).

\begin{tabular}{|c|c|c|}
\hline Method & Overall Accuracy (OA) & Kappa \\
\hline DT & $67.80 \%$ & $65.70 \%$ \\
\hline Boosted DT & $83.60 \%$ & $82.50 \%$ \\
\hline RF & $83.40 \%$ & $82.30 \%$ \\
\hline SVM-Radial & $87.80 \%$ & $87.00 \%$ \\
\hline KNN & $80.60 \%$ & $79.30 \%$ \\
\hline ANN & $18.30 \%$ & $12.80 \%$ \\
\hline
\end{tabular}

Table 3: Classification accuracies for each classification method.

The four algorithms with OA greater than $80 \%$ produced similar maps that were visually consistent with reality (i.e. coherent classes and minimal speckling) (Fig. 5) and represented the classification land cover for 10 classes: Forest, Grass, Water, Bushes, Bare soil, Urban area or built-up, Mining, Secondary forest, Clouds and Shadows.

A detailed visual analysis of land-cover classification methods employed and that obtained an OA greater than $80 \%$ was made. From that visual analysis, the boundary of the secondary forest, is better defined by land-cover classification based in SVMradial than the other three methods. The class mining is uniform in all the four methods but in the map obtained with SVM, exist a zone where the area of mining is distinguished between bushes and grassland, in some areas the shadows was confused with the class water for its similar spectral behavior, however spectral indices used for landcover classification helped distinguish between vegetation and soil types and minimize the 
influence of shadows. Due to the high spatial resolution and the spectral resolution of image fused it is possible to classify and separate built-up, bare soil and vegetation in urban areas. In general class boundaries in land-cover classification based on the fused imagery are more natural, realistic, and have better coincide with the reality mainly with the SVM-Radial algorithm.
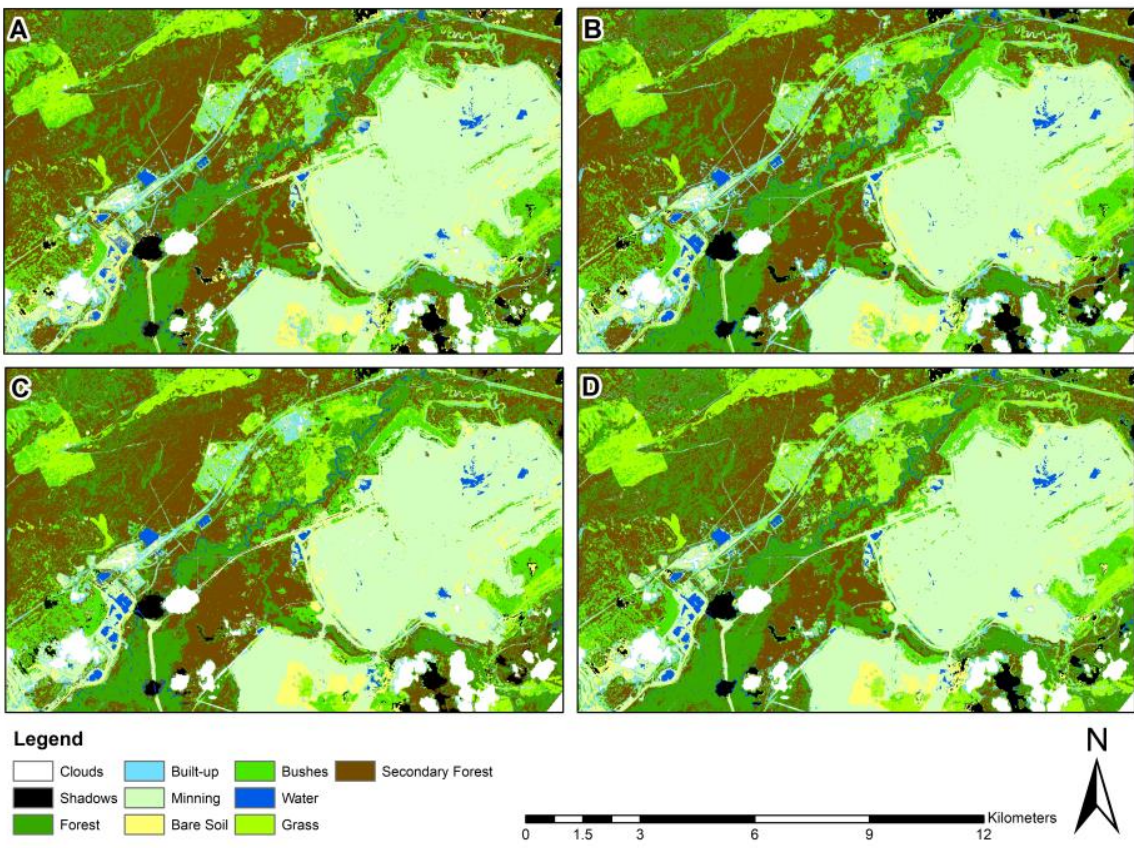

Figure 5: Final classified maps of the study area for methods with overall accuracy greater than $80 \%$. (A) Boosted Decision Tree (DT); (B) Random Forest (RF); (C) Support Vector Machine (SVM)-Radial; (D) K-Nearest Neighbors (KNN).

\section{CONCLUSIONS}

The focus of this research was to compare different machine learning algorithm for pixel classification of imagery fused from sensors Sentinel-2A and PlanetScope. The method used for image fusion was a variational model for $\mathrm{P}+\mathrm{XS}$; the machine learning algorithms assessment were six: DT (Decision Tree), Boosted DT, RF (Random Forest), SVM radial base (Support Vector Machine), ANN (Artificial Neural Networks), KNN (kNearest Neighbors). The results showed that the maps derived from four machine learning algorithms have similar accuracy, however SVM radial base has the highest accuracy (OA: 87.8\%, Kappa: $87 \%$ ) respect to the other methods (Boosted DT, DT, RF, ANN y KNN). Nevertheless, the methods RF, Boosted DT and KNN shown to be very powerful methods for classification of the study area. But as (Maxwell et al., 2018) indicate, the best algorithm for a specific task may be case-specific and may depend on the classes being mapped, the nature of the training data, and the predictor variables provided.

Using image fusion, a good classification was obtained, thanks to the high spectral resolution of Sentinel-2A for discriminating of the different classes and the high spatial resolution of PlanetScope for identifying terrain features and structures. This let to improve the classification of remote sensing images, used in applications as environmental, ecological, land-use among others. Therefore, the methods of machine learning and image fusion employed and presented in this paper can easily be applied to other studies.
The fusion imagery on land-cover classification let have a good delimitation in the classes of soil and vegetation like grassland, brushes and forest in pixel-based classifications, thanks to the high resolution reduced errors of omission in soil and low vegetation classes and reduced errors of commission in built-up and forest classes, normally presented in classifications used low resolution imagery. to limited training data, so a recommendation is having a larger set of training data for this algorithm and to achieve a better classification accuracy, the recommendation is that for future works to employ an ensembled model with the best models, it could achieve better prediction.

\section{ACKNOWLEDGEMENTS}

I would like to thank National Mining Agency of Colombia for providing the PlanetScope image used in the present study.

\section{REFERENCES}

Abdi, A. M. (2019). Land cover and land use classification performance of machine learning algorithms in a boreal landscape using Sentinel-2 data. GIScience \& Remote Sensing, O(0), 1-20. https://doi.org/10.1080/15481603.2019.1650447

Addesso, P., Conte, R., Longo, M., Restaino, R., \& Vivone, G. (2012). SVM-based cloud detection aided by contextual information. 2012 Tyrrhenian Workshop on Advances in Radar and Remote Sensing (TyWRRS), 214-221. https://doi.org/10.1109/TyWRRS.2012.6381132

Alparone, L., Wald, L., Chanussot, J., Thomas, C., Gamba, P., \& Bruce, L. M. (2007). Comparison of Pansharpening Algorithms: Outcome of the 2006 GRS-S Data-Fusion Contest. IEEE Transactions on Geoscience and Remote Sensing, 45(10), 3012-3021. https://doi.org/10.1109/TGRS.2007.904923

Ballester, C., Caselles, V., Igual, L., Verdera, J., \& Rougé, B. (2006). A Variational Model for P+XS Image Fusion. 
International Journal of Computer Vision, 69(1), 43-58. https://doi.org/10.1007/s11263-006-6852-x

Boulesteix, A.-L., Janitza, S., Kruppa, J., \& König, I. R. (2012). Overview of random forest methodology and practical guidance with emphasis on computational biology and bioinformatics. Wiley Interdisciplinary Reviews: Data Mining and Knowledge Discovery, 2(6), 493-507. https://doi.org/10.1002/widm.1072

Bradski, G., \& Kaehler, A. (2013). Learning OpenCV: Computer Vision in $\mathrm{C}++$ with the OpenCV Library (2nd ed.). O'Reilly Media, Inc.

Buddhiraju, K. M., \& Rizvi, I. A. (2010). Comparison of CBF, ANN and SVM classifiers for object based classification of high resolution satellite images. 2010 IEEE International Geoscience and Remote Sensing Symposium, 40-43. https://doi.org/10.1109/IGARSS.2010.5652033

Copernicus. (2017). Sentinel-2-Missions-Sentinel Online. Recuperado 5 de septiembre de 2019, de https://sentinel.esa.int/web/sentinel/missions/sentinel-2

Evrendilek, F., \& Gulbeyaz, O. (2011). Boosted decision tree classifications of land cover over Turkey integrating MODIS, climate and topographic data. International Journal of Remote Sensing, $32(12)$ 3461-3483. https://doi.org/10.1080/01431161003749469

Gašparović, M., Medak, D., Pilaš, I., Jurjević, L., \& Balenović, I. (2018). FUSION OF SENTINEL-2 AND PLANETSCOPE IMAGERY FOR VEGETATION DETECTION AND MONITORING. ISPRS - International Archives of the Photogrammetry, Remote Sensing and Spatial Information Sciences, XLII-1, 155-160. https://doi.org/10.5194/isprsarchives-XLII-1-155-2018

Gašparović, Mateo, \& Jogun, T. (2017). The effect of fusing Sentinel-2 bands on land-cover classification. International Journal of Remote Sensing, 39(3), 822-841. https://doi.org/10.1080/01431161.2017.1392640

He, X., Condat, L., Chanussot, J., \& Xia, J. (2012). Pansharpening using total variation regularization. 2012 IEEE International Geoscience and Remote Sensing Symposium, 166-169. https://doi.org/10.1109/IGARSS.2012.6351611

Jensen, J. R. (2005). Introductory Digital Image Processing: A remote sensing perspective (Tercera). United States of America: Pearson Practice Hall.

Kavzoglu, T., \& Colkesen, I. (2009). A kernel functions analysis for support vector machines for land cover classification. International Journal of Applied Earth Observation and Geoinformation, 11(5),

https://doi.org/10.1016/j.jag.2009.06.002

Keshtkar, H., Voigt, W., \& Alizadeh, E. (2017). Land-cover classification and analysis of change using machine-learning classifiers and multi-temporal remote sensing imagery. Arabian Journal of Geosciences, 10(6), 154 https://doi.org/10.1007/s12517-017-2899-y

Kuhn, M., Weston, S., Williams, A., Keefer, C., Engelhardt, A., Cooper, T., ... Wing, J. (2019). caret: Classification and Regression Training (Versión 6.0-84). Recuperado de https://CRAN.R-project.org/package=caret

Lizarazo, I. (2008). SVM-based segmentation and classification of remotely sensed data. International Journal of Remote Sensing, $29(24)$ $7277-7283$ https://doi.org/10.1080/01431160802326081

Lizarazo, Ivan. (2010). Fuzzy image regions for estimation of impervious surface areas. Remote Sensing Letters, 1(1), 19-27. https://doi.org/10.1080/01431160903246683

Maxwell, A. E., Warner, T. A., \& Fang, F. (2018). Implementation of machine-learning classification in remote sensing: An applied review. International Journal of Remote
Sensing,

$39(9)$,

2784-2817.

https://doi.org/10.1080/01431161.2018.1433343

Nunez, J., Otazu, X., Fors, O., Prades, A., Pala, V., \& Arbiol, R. (1999). Multiresolution-based image fusion with additive wavelet decomposition. IEEE Transactions on Geoscience and Remote Sensing, 37(3), 1204-1211. https://doi.org/10.1109/36.763274

Olofsson, P., Foody, G. M., Herold, M., Stehman, S. V., Woodcock, C. E., \& Wulder, M. A. (2014). Good practices for estimating area and assessing accuracy of land change. Remote Sensing of Environment, 148, 42-57. https://doi.org/10.1016/j.rse.2014.02.015

Otazu, X., Gonzalez-Audicana, M., Fors, O., \& Nunez, J. (2005). Introduction of sensor spectral response into image fusion methods. Application to wavelet-based methods. IEEE Transactions on Geoscience and Remote Sensing, 43(10), 2376-2385. https://doi.org/10.1109/TGRS.2005.856106

Petropoulos, G. P., Partsinevelos, P., \& Mitraka, Z. (2013). Change detection of surface mining activity and reclamation based on a machine learning approach of multi-temporal Landsat TM imagery. Geocarto International, 28(4), 323-342. https://doi.org/10.1080/10106049.2012.706648

PlanetLabs. (2019, abril 29). Cloud-based Satellite Imagery platform. Recuperado 5 de septiembre de 2019, de Planet website: https://planet.com/products/platform/

Qi, J., Chehbouni, A., Huete, A. R., Kerr, Y. H., \& Sorooshian, S. (1994). A modified soil adjusted vegetation index. Remote Sensing of Environment, 48(2), 119-126. https://doi.org/10.1016/0034-4257(94)90134-1

Renza Torres, D. (2010). IMPLEMENTACION $Y$ EVALUACION DE UN FILTRO DIGITAL PARA LA TRANSFORMADA WAVELET COMPLEJA DE DOBLE ARBOL (DT-CWT) APLICADA A FUSION DE IMAGENES BIDIMENSIONALES EN ESCALA DE GRIS. Universidad Nacional de Colombia, Bogota.

Schneider, A. (2012). Monitoring land cover change in urban and peri-urban areas using dense time stacks of Landsat satellite data and a data mining approach. Remote Sensing of Environment, 124 , 689-704. https://doi.org/10.1016/j.rse.2012.06.006

Shih, H., Stow, D. A., \& Tsai, Y. H. (2018). Guidance on and comparison of machine learning classifiers for Landsat-based land cover and land use mapping. International Journal of Remote Sensing, 40(4), 1248-1274. https://doi.org/10.1080/01431161.2018.1524179

Sivagami, R., Krishankumar, R., \& Ravichandran, K. S. (2018). A Comparative Analysis of Supervised Learning Techniques for Pixel Classification in Remote Sensing Images. 2018 International Conference on Wireless Communications, Signal Processing and Networking (WiSPNET), 1-4. https://doi.org/10.1109/WiSPNET.2018.8538518

Tan, P.-N., Steinbach, M., Karpatne, A., \& Kumar, V. (2018). Introduction to Data Mining (2Nd Edition) (2nd ed.). Pearson.

Tang, Y., Jing, L., Atkinson, P. M., \& Li, H. (2016). A multiplepoint spatially weighted k-NN classifier for remote sensing. International Journal of Remote Sensing, 37(18), 4441-4459. https://doi.org/10.1080/01431161.2016.1214300

Tso, B., \& Mather, P. (2016). Classification Methods for Remotely Sensed Data. https://doi.org/10.1201/9781420090741

Vallauri, D., Aronson, J., Dudley, N., \& Vallejo, R. (2005). Monitoring and Evaluating Forest Restoration Success. En S. Mansourian, D. Vallauri, \& N. Dudley (Eds.), Forest Restoration in Landscapes: Beyond Planting Trees (pp. 150158). https://doi.org/10.1007/0-387-29112-1_21 
The International Archives of the Photogrammetry, Remote Sensing and Spatial Information Sciences, Volume XLII-3/W12-2020, 2020 2020 IEEE Latin American GRSS \& ISPRS Remote Sensing Conference (LAGIRS 2020), 22-26 March 2020, Santiago, Chile

Wald, L. (1999). Some terms of reference in data fusion. IEEE Transactions on Geoscience and Remote Sensing, 37(3), 1190-1193. https://doi.org/10.1109/36.763269

Wang, Z., \& Bovik, A. C. (2002). A universal image quality index. IEEE Signal Processing Letters, 9(3), 81-84. https://doi.org/10.1109/97.995823

Wilson, E. H., \& Sader, S. A. (2002). Detection of forest harvest type using multiple dates of Landsat TM imagery. Remote Sensing of Environment, 80(3), 385-396. https://doi.org/10.1016/S0034-4257(01)00318-2

Wu, J., Wang, D., \& Bauer, M. E. (2007). Assessing broadband vegetation indices and QuickBird data in estimating leaf area index of corn and potato canopies. Field Crops Research, 102(1), 33-42. https://doi.org/10.1016/j.fcr.2007.01.003

Zha, Y., Gao, J., \& Ni, S. (2003). Use of normalized difference built-up index in automatically mapping urban areas from TM imagery. International Journal of Remote Sensing, 24(3), 583594. https://doi.org/10.1080/01431160304987 